\title{
Optimal Design of Switched Reluctance Motor Using PSO Based FEM-EMC Modeling
}

\author{
Mouellef Sihem, Bentounsi Amar, Benalla Hocine \\ LGEC, Laboratory of Electrotechnics Dept., Mentouri University of Constantine 1, Algeria
}

\author{
Article Info \\ Article history: \\ Received Mar 25, 2015 \\ Revised Jun 3, 2015 \\ Accepted Jun 25, 2015

\section{Keyword: \\ Equivalent Magnetic Circuit \\ Finite Element Method \\ Genetic Algorithms \\ Particle Swarm Optimization \\ Switched Reluctance Motor}

\begin{abstract}
This paper aims to optimize the design of a prototype of a 6/4 Switched Reluctance Motor (SRM) using the Particle Swarm Optimization (PSO) algorithm. The geometrical parameters to optimize are the widths of the stator and rotor teeth due to their significant effects on the prototype design and the performances in terms of increased average torque and reduced torque ripple. The studied 3kW SRM is modeled using a numerical-analytical approach based on a coupled Finite Element Method with Equivalent Magnetic Circuit (FEM-EMC). The simulations are performed under MATLAB environment with user-friendly software. The optimal results found are discussed, compared against those obtained by the Genetic Algorithms (GA) and showed a significant improvement in average torque.
\end{abstract}

Copyright () 2015 Institute of Advanced Engineering and Science. All rights reserved.

\section{Corresponding Author:}

Mouellef Sihem,

LGEC, Laboratory of Electrotechnics Dept.,

Mentouri University of Constantine 1,

47 Rue E-A-K, Khroub, Constantine, Algeria.

Email: mouellef_sihem@yahoo.fr

\section{INTRODUCTION}

To meet challenging requirements, new design and more efficient structures of electrical machines are investigated by manufacturers and researchers. In this context, Permanent Magnet Synchronous Motor (PMSM), Brushless dc motor (BLDC), Linear Switched Reluctance Motor (LSRM) and rotary Switched Reluctance Motors (SRMs) have been explored in the literature as they are an attractive alternative to induction and synchronous machines [1-4]. Due to their robustness, reliability, high performance and reduced cost, the SRM found numerous applications at high speed drive or low speed generator (electrical vehicles, air-conditioners, extractors, centrifuges, flywheel energy storage, shipbuilding, aeronautics, and gearless wind generator) [5-7].

Metaheuristic methods are general optimizing algorithms applicable to a wide variety of problems. They appeared in the 1980s, with a common ambition: to solve efficiently the difficult optimization problems, for which there is no known most effective classical method [8], [9]. New techniques inspired by artificial intelligence have emerged and developed to offer as potential alternative techniques to improve the quality of the solution, namely Genetic Algorithms (GA), Particle Swarm Optimization (PSO), and so on.

The PSO is a still relatively unknown and relatively young technique in the field of design [10], [11]. It is analogous to GA in the sense that the system is initialized with a random population of solutions; it is compared to all its neighbors by maintaining each time the best result [12]. Unlike the GA and other metaheuristic algorithms, PSO has the flexibility to control the balance between global and local exploration of the search space [13], [14]. The PSO has achieved rapid development following advantages [15-17]: simple concept, easy implementation, robustness and computational efficiency. In [18], the torque production is improved using PSO algorithm to optimize the stator and rotor angles of a 8/6 SRM. In [19], the PSO is applied to the rotor pole arc of a 4/2 SRM to minimize the torque ripple. 
One aspect of the contribution of this work lies with the application of the Particle Swarm Optimization method (PSO) for optimizing the average torque of a 6/4 SRM through various geometric parameters. The other aspect is the comparative study of the performance of PSO and GA algorithms applied to the machine GA [20]. The results show that the PSO-based approach gives the best performance in terms of solution quality, accuracy and convergence time. The main contribution of this work is related to the numerical-analytical approach used to model the studied SRM using a user-friendly program carried out under MATLAB.

The paper is organized as follows: Section 2 describes the FEM-EMC approach modeling of the studied SRM. In Section 3 the PSO algorithm is presented with the formulation of the problem. The results obtained are discussed in Section 4. The paper concludes in Section 5.

\section{FEM-EMC MODELING OF THE SRM}

Modeling of electric machines can be classified into three categories: analytical models, finite element analysis (FEA) and equivalent magnetic circuits (EMC), which can be considered as a semianalytical method [21]. Modeling using EMC has been chosen for further investigation because it seemed a good technique with great speed and acceptable accuracy. The model produced will be used later in an optimization process that aims to find the best system parameters.

The machine topology studied is a double saliency three-phase 6/4 SRM with Ns=6 stator teeth and $\mathrm{Nr}=4$ rotor teeth as represented "Figure 1". Its operating principle, similar to the stepper motor, has long been known: by exciting successively the three stator phases, the rotor teeth are positioned to maximize the inductance of the power phase, under the rule of 'maximum flux' (aligned position); by turning off the power, the motor will continue its movement until it reaches a position corresponding to the minimum value of inductance or flux (unaligned position). On the linked flux ( $\lambda$ )-current ( $i$ ) characteristics, the area between the previous two extreme positions represents the electrical energy converted into mechanical energy per cycle, $\mathrm{W}=\mathrm{W}_{\mathrm{a}}-\mathrm{W}_{\mathrm{u}}$, as shown in "Figure 2 ".

As described in [22], to determine analytically the relations flux-At from only seven characteristics equal-flux lines traced by the finite element method (FEM) and corresponding to seven magnetic equivalent circuits (EMC), we implemented a program in MATLAB package software for the iterative calculation of the saturated aligned and unaligned inductances, respectively $L_{a}$ and $L_{u}$, and the corresponding energies, $W_{a}$ and $W_{u}$, from which one can deduce the average torque as depicted in "Figure 3":

$$
\begin{aligned}
& T_{a v}=\frac{q N_{r}\left(W_{a}-W_{u}\right)}{2 \pi} \\
& W_{a}=\left(\lambda_{1}+\lambda_{2}+\ldots \ldots \ldots+\frac{1}{2} \lambda_{n}\right) * \delta i \\
& W_{u}=\frac{1}{2} \lambda_{u} I_{p} \\
& \delta_{i}=\frac{I_{p}}{n}
\end{aligned}
$$

The integration step $\delta_{i}$ is the ratio of the peak value of current $I_{p}$ on the number of intervals $n$.

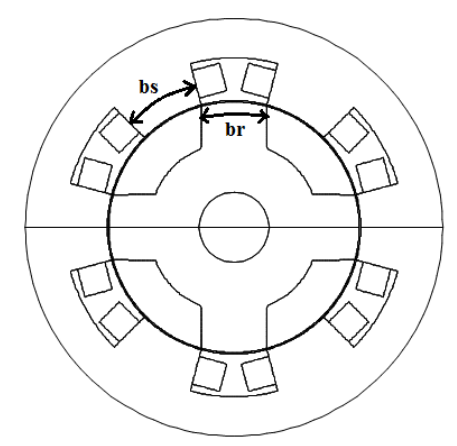

Figure 1. Cross-section of the studied 6/4 SRM 


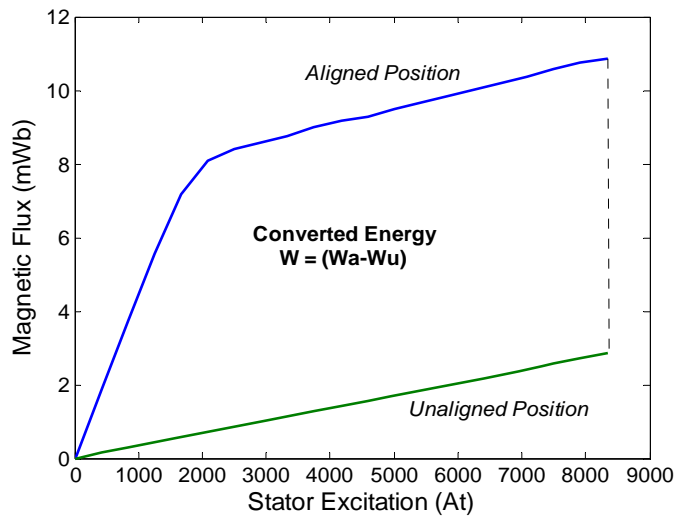

Figure 2. Extremes magnetic characteristics flux vs. excitation mmf

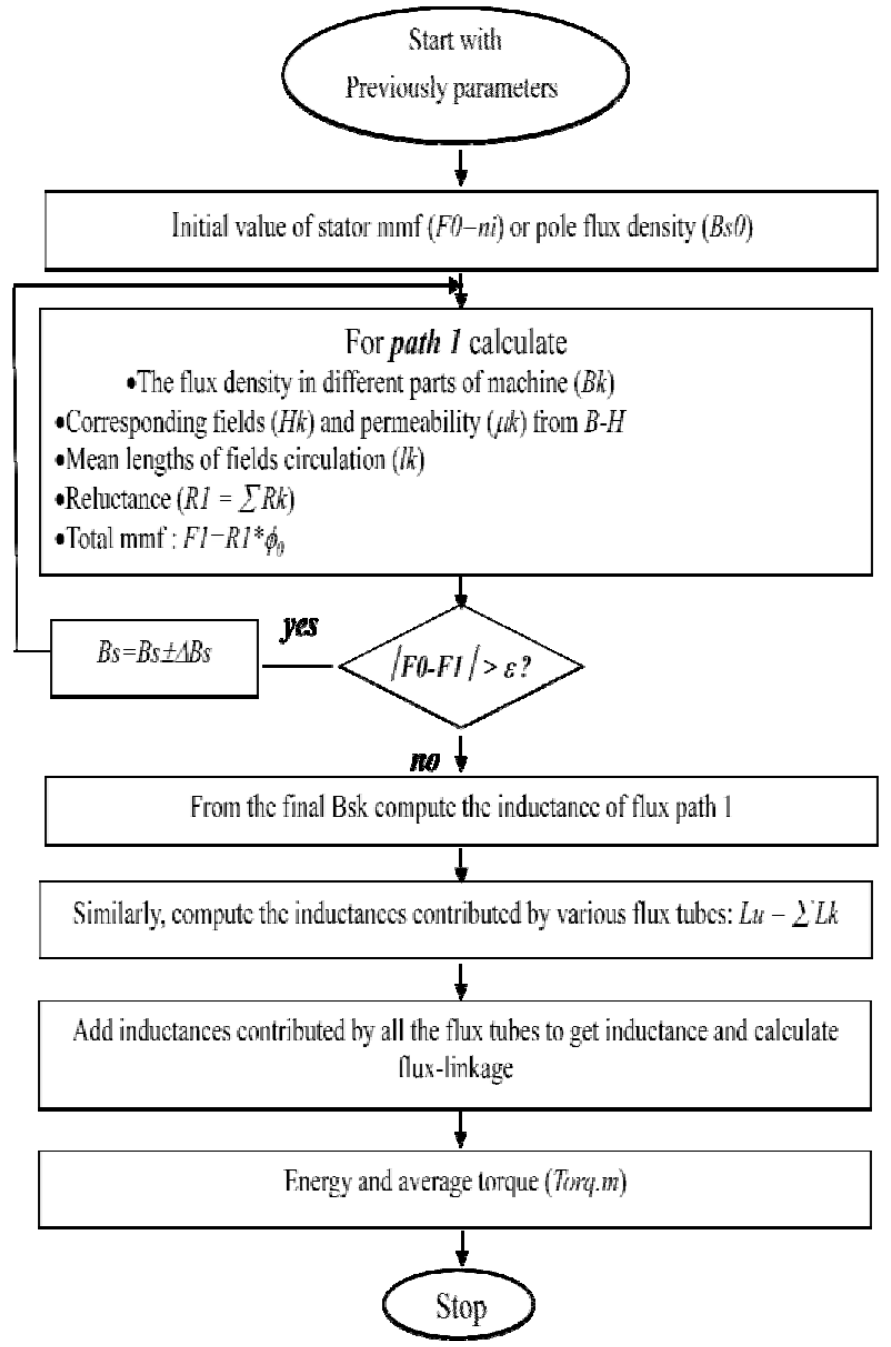

Figure 3. Flowchart of main program of simulation under MATLAB 


\section{PARTICLE SWARM OPTIMIZATION ALGORITHM}

\subsection{Basic Concepts of PSO}

The Particle Swarm Optimization method (PSO) is a relatively recent heuristic proposed by Eberhart and Kennedy for the first time in the early 90s [23], based on a stochastic population candidates solutions to develop an optimal solution to the problem presented. This method is particularly suitable for non-linear systems; it does not require the calculation of the first and second derivative, unlike the gradient type methods. Its basic idea is inspired from the actions of animal groups (swarms) in their search for the best subsistence areas. Thus, each individual in the population has the memory of its previous experience and the information provided by the group on the most promising regions. This contribution to the overall experience, in addition to personal experience is one of the features of PSO which ensure it success in global searches.

A swarm of particles, which are potential solutions to the optimization problem, "flies" the search space, in the search for the global optimum. The movement of a particle is influenced by three components [24]:

1. A component of inertia: the particle tends to follow its current travel direction;

2. A cognitive component: the particle tends to rely on its own experience, and thus to move towards the best site in which it has already passed;

3. A social component: the particle tends to rely on the experience of its congeners, and thus to move towards the best sites already reached collectively by the swarm.

\subsection{Basic Principle of PSO}

In a search space of dimension $D$, the algorithm starts with a random initialization of the particle swarm. Particle i of the swarm is modeled by its position vector $\vec{x}_{i}=\left(x_{i 1}, x_{i 2}, \ldots, x_{i D}\right)$ and the velocity vector $\vec{v}_{i}=\left(v_{i 1}, v_{i 2}, \ldots, v_{i D}\right)$.

The quality of its position is determined by the value of the objective function at that point. This particle remembers the best position in which it has already passed, which is noted $\vec{P}$ best $_{i}=\left(\right.$ pbest $_{i 1}$, pbest $_{i 2}, \ldots$, pbest $\left._{i D}\right)$. The best position achieved by its neighboring particles is noted $\vec{G}$ best $=\left(\right.$ gbest $_{1}$, gbest $_{2}, \ldots$, gbest $\left._{D}\right)$.

Indeed, at iteration $t+1$, the velocity vector and the position vector are calculated from the equation (5) and equation (6), respectively.

$$
\begin{aligned}
& v_{i, j}^{t+1}=w v_{i, j}^{t}+c_{1} r_{1, j}^{t}\left[\text { pbest } t_{i, j}-x_{i, j}^{t}\right]+c_{2} r_{2, j}^{t}\left[\text { gbest }-x_{i, j}^{t}\right] \\
& x_{i, j}^{t+1}=x_{i, j}^{t}+v_{i, j}^{t+1}, j \in\{1,2, \ldots ., D\}
\end{aligned}
$$

where $: v_{i, j}^{t+1}, v_{i, j}^{t}$ : Are the speed of the particle to $\mathrm{t}$ and $\mathrm{t}+1$ iterations. Pbest : Is the best position of the particle. Gbest: Is the best position of a neighbor at iteration t. $x_{i, j}^{t}$ : Is the position of the particle at iteration t. $w$ : Is generally called a constant factor of inertia, it keeps a balance between exploration and exploitation. $C_{1}$ and $C_{2}$ : are two constants called acceleration coefficients, they keep the balance between individual and social behavior of the particle when they are equal [25]. $r_{1}$ and $r_{2}$ : are two randomly generated numbers with a range of $[0,1]$, for each iteration and for each dimension $j$.

\subsection{Problem Formulation}

The objective function $f(x, u)$ used to formulate the SRM problem represents a maximizing average torque. In the case of an optimization problem where the objective is to be maximized, the function is considered with the opposite sign $-f(x, u)$; the equality constraints expressed by the function $g(x, u)$ are represented by the equations of the maximum and minimum inductances $L_{a}$ and $L_{u}$ as well as $W_{a}$ and $W_{u}$ energies of the two extreme positions of which the average torque and inequality constraints will be deduced which reflect the lower and upper dental openings which are given by equations limits [26]: 


$$
\begin{aligned}
& \beta_{s m}=\left(2 \pi / q N_{r}\right)=30^{\circ} \leq \beta_{s} \leq 45^{\circ}=\left(\pi / N_{r}\right) \\
& \beta_{r m}=\beta_{s m}=30^{\circ} \leq \beta_{r} \leq 60^{\circ}=\left(\alpha_{r}-\beta_{s m}\right) \\
& \left(\beta_{s}+\beta_{r}\right)<a_{r}=\left(2 \pi / N_{r}\right)=90^{\circ}
\end{aligned}
$$

Therefore, the proposed solutions must take the constraints of construction into account. These constraints are taken into account by penalizing proportionally the objective function for constraint violations. In the context of taking into account the constraints, it is to degrade the performance of infeasible individuals in function of their proximity to the feasible area of the search space. For each element of the search space, its proximity to the feasible region can be measured through the level of violation of each constraint. Using this measure of infeasibility of the individual $x$ from each constraint, the penalty function in the general form can be introduced:

$$
\begin{aligned}
& \operatorname{Min} F_{o b j}=-f(x)+F_{\text {penalty }} \\
& x_{i}^{1} \leq x_{i} \leq x_{i}^{u}, i=1, \ldots \ldots, n
\end{aligned}
$$

\section{SIMULATION RESULTS AND DISCUSSION}

A comparative study with Genetic Algorithms (GA) has been made to verify the performance of the proposed algorithm.

The PSO and GA parameters used for simulation are summarized in Table 1. For the implementation of PSO, several parameters must be specified, such as acceleration factors ( $C_{1}$ and $C_{2}$ ), the inertia factor $(w)$, the size of the swarms and the stop criterion. The PSO algorithm has been applied to the objective function according to the flowchart in Figure 4.

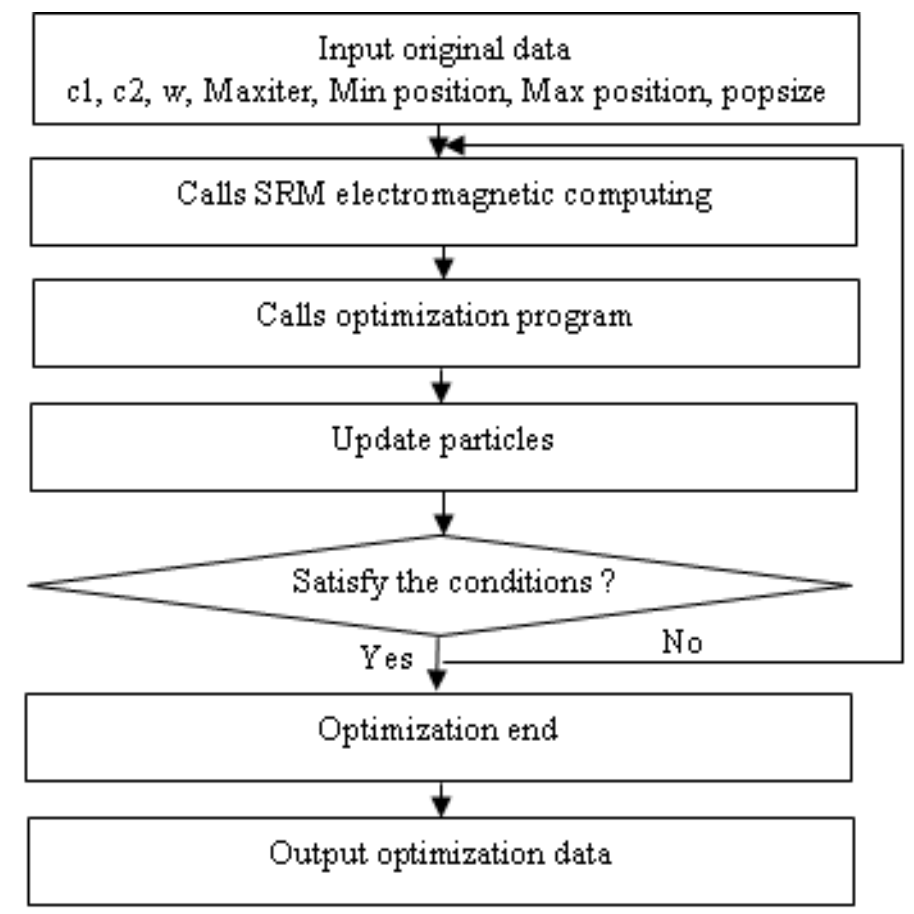

Figure 4. The flowchart of adaptive PSO for SRM 
Table 1. Simulation parameters

\begin{tabular}{cc}
\hline GA & PSO \\
\hline Population size: 20 & Number of Particles:20 \\
Generations:100 & Iterations:100 \\
Crossover rate: 0.7 & $C_{1}=C_{2}=2$ \\
Mutation rate:0.09 & $W=-0.1$ \\
\hline
\end{tabular}

To confirm the performance of this method, a comparison of its results with the results of genetic algorithms has been made. The comparison is shown in Table 2.

The convergence characteristics of the two methods: PSO and GA for a variable reluctance motor are shown in Figures 5, 6, and 7. According to the results, it can be noticed that the PSO explores a solution superior to the genetic algorithm for the same number of population and generation.

Table 2. Comparison results of PSO and GA for $\mathrm{Fa}=1691 \mathrm{At}$

\begin{tabular}{cccc}
\hline parameters & Prototype & GA & PSO \\
\hline Stator pole arc (deg.) & 30 & 39.9409 & 37.8324 \\
Rotor pole arc (deg.) & 30 & 49.9333 & 52.1676 \\
average torque (Nm) & 11.1399 & 15.543 & 15.6178 \\
\hline
\end{tabular}

It can be seen from figure 5, firstly, that the PSO algorithm converges toward the global optimum from the thirty sixth iteration (36), while the convergence of the GA algorithm is reached at iteration (26) with an optimal value lower compared to the PSO algorithm. This proves that the power of convergence to the global optimum in the PSO method exceeds that of the method of genetic algorithms (GA), this will have a direct impact on the time required for convergence of the two methods. Furthermore, the robustness of the PSO algorithm is more remarkable. The difference in average torque between the two optimization methods (15.6178Nm compared with $15.543 \mathrm{Nm}$ ) is virtually insignificant or negligible (a slight difference of about $0.48 \%)$. This will confirm our findings in terms of robustness of the PSO convergence.

The results presented in Figures 6 and 7 show variations of $\beta_{s}$ and $\beta_{r}$ around their optimum values. The safety constraints are also checked for these two angles. These are qualified in their ranges.

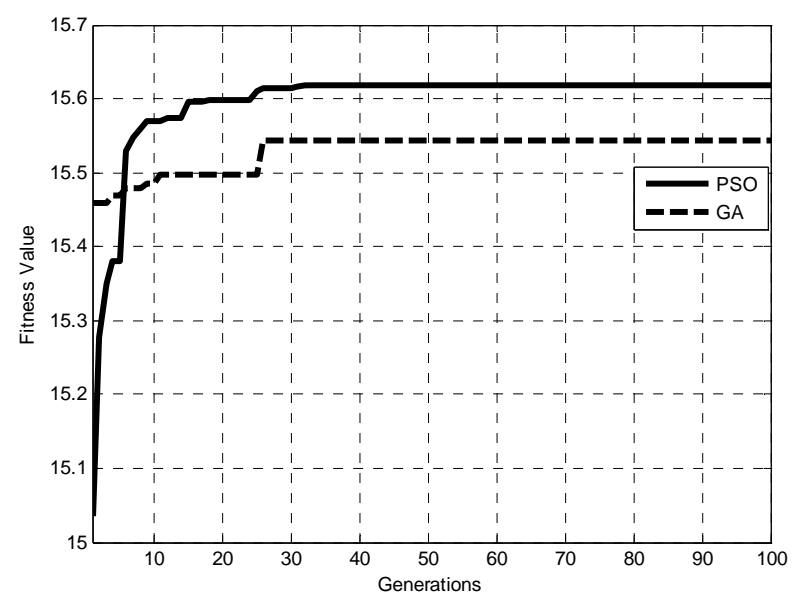

Figure 5. Objective function 


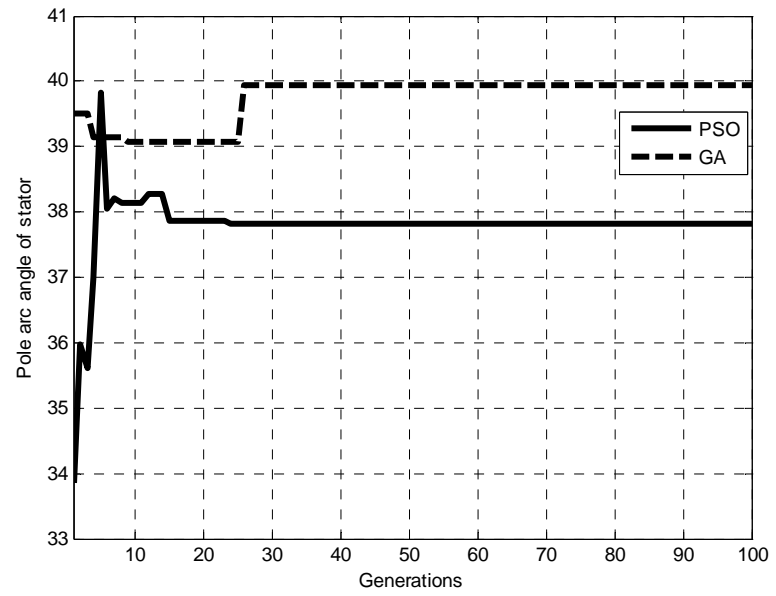

Figure 6. Change of $\beta_{s}$ around the optimal value

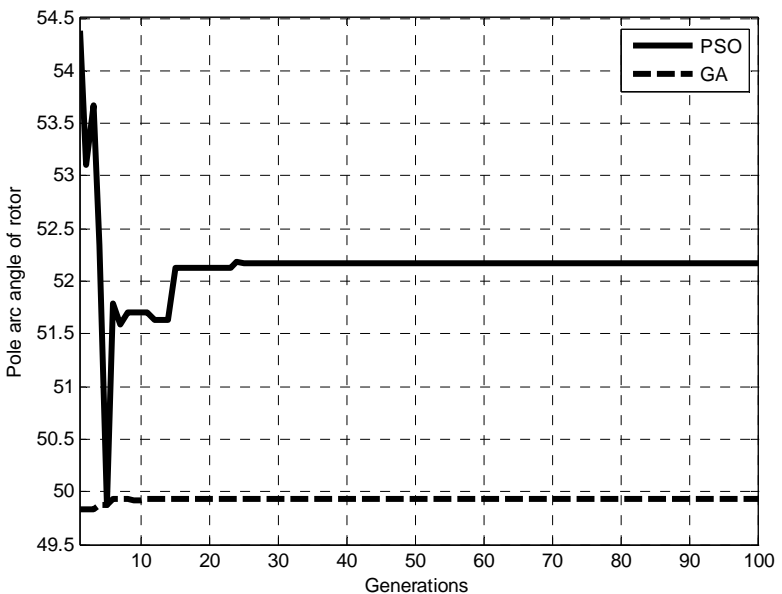

Figure 7. Change of $\beta_{r}$ around the optimal value

\section{CONCLUSION}

The paper proposes a permeance network modeling and presents a direct coupling between the finite element method (FEM) and the equivalent magnetic circuit method (EMC) to model the switched reluctance machine. The modeling tool presented is designed to be integrated into an optimization process that modifies the geometry of the engine.

The optimization method chosen is the particle swarm optimization (PSO), the stochastic nature, metaheuristics, allows the application to difficult and non-linear problems. The principle of the method is explained as well as the different coefficients of the algorithm and the influence they have on the evolution of the algorithm. The optimization procedure of the design of SRM using PSO is presented with the aim of maximizing the average torque for an efficient solution all by acting on the tooth geometry which has a great influence on motor performance. The difference in the average torque estimated by the two algorithms, PSO and GA, is negligible suggesting the simultaneous convergence to the same quasi-optimal solution.

From the simulation results, it can be found that the PSO can lead to optimal feasible solution, and that is the relative ease of implementation and ability to provide reasonably good solutions for many combinatorial problems.

\section{REFERENCES}

[1] S. Lakshmikanth, K.R. Natraj and K.R. Rekha. "Noise and Vibration Reduction in Permanent Magnet Synchronous Motors-A Review". International Journal of Electrical and Computer Engineering. 2012; 2(3): 405-416. 
[2] H.M. Soliman and S.M.EL. Hakim. "Optimum Remedial Operation of Permanent Magnet Synchronous Motor". International Journal of Electrical and Computer Engineering. 2012; 2(5): 621-631.

[3] T. Raghu, S. Chandra Sekhar and J. Srinivas Rao. "SEPIC Converter based-Drive for Unipolar BLDC Motor". International Journal of Electrical and Computer Engineering. 2012; 2(2): 159-165.

[4] N.C. Lenin and R. Arumugam. "Design and Experimental Verification of Linear Switched Reluctance Motor with Skewed Poles". International Journal of Electrical and Computer Engineering. 2015; 6(1): 18-25.

[5] M. Zeroualia, M.E.H. Benbouzid and D. Diallo. "Electric Motor Drive Selection Issues for HEV Propulsion Systems: A Comparative Study". IEEE Trans. On Vehicular Technology. 2006; 55(6): 1756-1764.

[6] K.M. Rahman et al. "Advantages of Switched Reluctance Motor Applications to EV and HEV: Design and Control Issues". IEEE Trans. On Industry Applications. 2000; 36(1): 111-121.

[7] R. Cardenas et al. "Control of a Switched Reluctance Generator for Variable-Speed Wind Energy Applications". IEEE Trans. On Energy Conversion. 2005; 20(4): 781-791.

[8] Y. Colletto and P. Siarry. "Optimisation Multi-objectif". EYROLLES. Nov. 2002.

[9] M. Clerc and P. Siarry. "Une nouvelle métaheuristique pour l'optimisation difficile: la méthode des essaims particulaires". J3eA. 3 (7), 2004.

[10] J. Kennedy. "The Particle Swsrrm Socist Adaptation of Knowledge". Proceedings of the 1997 IEEE international Conference on Evolutionary Computation ICEC'97, Indianapolis, Indiana, USA. 1997, pp. 303-308.

[11] Y. Shi and R. Eberhart. "A modified particle swarm optimizer". in The 1998 IEEE International Conference on Evolutionary Computation Proceedings, The 1998. Piscataway, NJ: IEEE World Congress on Computational Intelligence. 1998, pp. 69-73.

[12] R.C. Eberhart and Y. Shi. "Comparing inertia weights and constriction factors in particle swarm optimization". in: Proc. of the IEEE Congress on Evolutionary Computation, IEEE, Piscataway, San Diego, CA. 2000, pp. 84-88.

[13] R. Hassan and B. Cohanim, O. Weck. "A comparison of particle swarm optimization and the genetic algorithm". American Institute of Aeronautics and Astronautics. 2004.

[14] R. C. Eberhart and Y. Shi. "Comparison between genetic algorithms and particle swarm optimization". in Proc., IEEE international conference evolutionary computer. May 1998, pp 611-616.

[15] M. A. Abido. "Particle Swarm Optimization for Multi-machine Power System Stabilizer Design". 0-7803-71739/01/2001 IEEE

[16] M. Balaji and V. Kamaraj. "Particle Swarm Optimization Approach for Optimal Design of Switched Reluctance Machine". American Journal of Applied Sciences. 2011; 8(4): 374-381.

[17] W. Zhang and Y. Liu, and M. Clerc. "An adaptive PSO algorithm for reactive power optimization". in Proc. 6th Int. Conf. Advances in Power System Control, Operation and Management. Nov. 2003, pp. 302-307.

[18] M. Blaji and V. Kamaraj. "Design Optimization of SRM using PSO". in: 1st Int. Conf. on Electrical Energy System (ICEES), 3-5 Jan. 2011, Newport Beach CA, pp. 164-169.

[19] W. Phuangmalai and N. Chayopitak. "A Design Study of a 4/2 SRM Using PSO". in: 9th Int. Conf. ECTI-CON, 1618 May 2012, Phetchaburi.

[20] S. Mouellef, A. Bentounsi and H. Benalla. "Teeth Shape Design of a SRM for High Torque Using Genetic Algorithms”. Int. Journal of Scientific \& Engineering Research (2229-5518). Vol. 3, Issue 10, October 2012, pp. 1128-1132.

[21] Moallem, M. and G.E. Dawson."An improved magnetic equivalent circuit method for predicting the characteristics of highly saturated electromagnetic devices". IEEE Trans. Magnetics. 34: 3632-3635. DOI: 10.1109/20.717858

[22] R. Krishnan. "Switched Reluctance Motor Drives-Modeling, Simulation, Analysis, Design, and Applications". CRC Press. 2001.

[23] J. Kennedy and R. Eberhart. "Particle swarm optimization". in Proc., IEEE international conference on neural networks (ICNNN ’95), Vol. IV, Perth, Australia. 1995, pp 1942-1948.

[24] Abbas EL DOR. "Perfectionnement des algorithmes d'Optimisation par Essaim Particulaire.Applications en segmentation d'images et en électronique". Thèse de doctorat en informatique, université paris-est. 5 décembre 2012.

[25] R. Eberhart and J. Kennedy. "A new optimizer using particle swarm theory". in Proc. 6th Int. Symp. Micro Machine Human Sci. (MHS’95). 1995, pp. 39-43.

[26] P.J. Lawrenson et al. "Variable-speed SRM’s". IEE Proc. Vol. 127, Pt. B, No. 4, July 1980, pp. 253-265.

\section{BIOGRAPHIES OF AUTHORS}

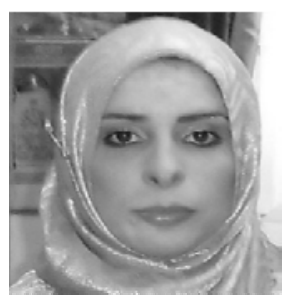

S. Mouellef received the M.S. degrees from Mentouri University of Constantine 1 . She is currently Assistant researcher in the Department of Electrical Engineering at the University of Constantine 1, Algeria. She is currently preparing his $\mathrm{PhD}$ degree in Constantine University, His research activities focus on the optimization system using methaeuristique methods. She has worked toward the development of the switched reluctance machine. 


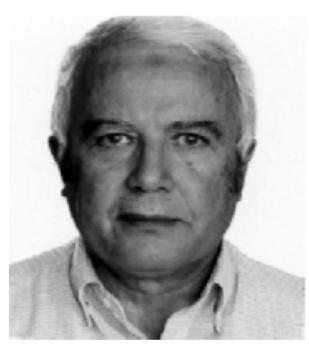

A. Bentounsi : (1953) After receiving its "doctorate-engineer" in Paris 6, France, in 1980, he joined the University of Constantine 1, Algeria, in 1984, as an Associate Professor. Since 1995, he is working on his Ph.D. dissertation in collaboration with the Cegely Lab. of Ecole Centrale, Lyon, France. Professor Bentounsi is the director of the Labo. de Génie Electrique de Constantine (LGEC), Algeria. His current research interests are CAD of the electrical machines and renewable energy conversion.

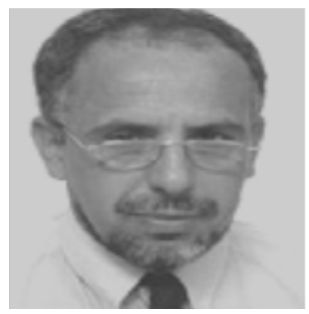

H. Benalla: (1957) He received the B.S., M.S., and Doctorate Engineer degrees in power electronics, from the National Polytechnic Institute of Toulouse, France, respectively in 1981, 1984. In 1995, he received the Ph.D. degree in Electrical Engineering from University of Jussieu-Paris VI, France. Since 1996, he is with the department of Electrotechnics, at Constantine University Algeria, as a Professor. His current research field includes Active Power Filters, PWM Inverters, Electric Machines, and AC Drives. 